\title{
Value of Reconstruction Procedures [non-microsurgical] on Facial Defects Postexcision of a Basocellular Carcinomas
}

\author{
${ }^{1}$ Faculty of Medicine, "Ovidius" University of Constanta \\ 2 Faculty of Medicine, "Ovidius" University of Constanta, Discipline - Anatomy \\ ${ }^{3}$ M.D. - dermatology \\ ${ }^{4}$ Faculty of Medicine, "Ovidius" University of Constanta, Discipline - Plastic Surgery \\ ${ }^{5}$ Faculty of Medicine, "Ovidius" University of Constanta, Discipline - Plastic Surgery
}

\begin{abstract}
The authors present a retrospective study - 2011-1014 on value of reconstructive nonmicrosurgicals procedures after excisions of facial basocellular carcinomas.

The study group included 426 cases of basocellular carcinomas of the face (primary diagnosed or recurrent) that required reconstructive procedure after excision in oncology margin (according to the criteria of the clinic). The choice is eclectically considering reconstructive process (evaluating) a number of factors: patient age, location of carcinoma, post excisional defect size and pathological status (recurrence, radiation) and the frequency of the primary outcome was using plastic reconstruction of full-thickness free skin.

All cases included in the study received a performant dermatoscopic exam and excisions were performed at 5-10 $\mathrm{mm}$ from the edges of the apparent surface and in deepness in fascial plane.
\end{abstract}

Keywords: face, basocellular carcinomas, reconstruction procedures.

\section{Iordache I.V.}

1st December1918 Boulevard, No. 37, Block L21, Ap. 15, Constanta Romania

iordacheionutvalentin@gmail.com

$+40721000052$

\section{Introduction}

Because visual exposure as the major role (decisive) in shaping of the physiognomy, face is the body region where the defects are hard to hide and the patient's expectations are much higher (in terms of cosmetic) than in other regions reconstruction.

Face and neck anatomy is complex and facial skin is directly related with facial muscles, providing expressiveness and specific features and complexity of anatomical structures is difficult to replicate: pyramid nose, lips, ear pavilion [1].

Facial reconstruction follows the same basic principles reconstructive:

- excised tissue to be replaced by similar one (usually local tissues provides the best match)

- reconstruction should not interfere with other healing treatments (radiotherapy, chemotherapy);

- simplicity of surgical intervention is not always the best solution [2];

- the defect coverage and functional restoration can be accomplished with one or more local flaps;

- the skin defects coverage has numerous solutions;

- quality of repair depends on the surgeon's 
surgical skills and ability to select the surgical procedure [3].

If all these criteria can be solved by using a local flap, it is preferable this to avoid the risks of microsurgical transfer or using a free skin transplant (for inconvenience of aesthetic reason) [4].

\section{Material and Method}

The present study aims to:

-realization a comprehensive medical record of the patient himself with basocellular carcinomas of the face that received non microsurgical post excisional reconstruction between 2011-2014 in surgery clinic of plastic and reconstructive microsurgery SCJU Constanta;

-to the main techniques used in the clinic based facial aesthetic subunit who underwent excision;

-formulation of a reconstruction algorithm based on fault location post excisional functional and aesthetic needs of the receiving area.

The study included all cases that required excisions for the formation of malignant tumor of the face - basocellular carcinomas -, which imposed a reconstruction procedure.

Mentioned that all malignant tumors included in the study were the basocellular skin tumor which, according to the clinic protocol, excision of the area was made at a distance of 5 to $10 \mathrm{~mm}$ from the edge of the apparent lesion and in profound level to coating fascia, applying the principle (rule) - " larger tumor, wider excision margins" [5].

In principle, protocol of the clinic use to cover post excisional defect with free autologous skin (full thickness free skin or free split skin) in order to be able of following over time easier occurrence of any relapses; there are also situations that require regional flaps and whenever possible direct suture excision margins even if it is done induce (at least initially) minimum local deformations.

In the study group were not included carcinomas lips these type require a specific post excisional reconstruction procedures, here carcinoma occurs more frequently spinocelular which requires specific surgical solutions (lymph dissection).

All cases included in the study received preoperative examination dermatoscope performance and achieved a diagnostic concordance with histopathology of over $98 \%$.

When is required usage of free autologous skin to cover the post excisional defect the clinic has in protocol (for the face) usage with priority of full-thickness free skin transplant having as favorite areas of harvesting: the internal face of the arm, retro auricular region, or abdomen in the presented order, and using a cutaneous free split skin transplantation only when the defect is very large. The solution of full thickness skin transplantation is the prevailing aesthetic aspects.

\section{Results}

This study is a retrospective one which is spanning in the interval 01.01.2011 - 31.12.2014 (respectively a period of four calendar years) and comprises of 426 hospitalized cases diagnosed and treated in plastic surgery and reconstructive microsurgery clinic of the Constanta County Emergency Hospital.

In the period January 2011 - December 2014 were hospitalized in the Department of Plastic Surgery and Burns Emergency County Hospital Constanta a number of 426 patients diagnosed with basocellular carcinomas located in the facial region.

The distribution of patients on years was as follows:

$$
\begin{aligned}
& \text { » Year } 2011=82 \text { patients } \\
& \text { »Year } 2012=94 \text { patients } \\
& \text { "Year } 2013=114 \text { patients } \\
& \text { »Year } 2014=136 \text { patients }
\end{aligned}
$$




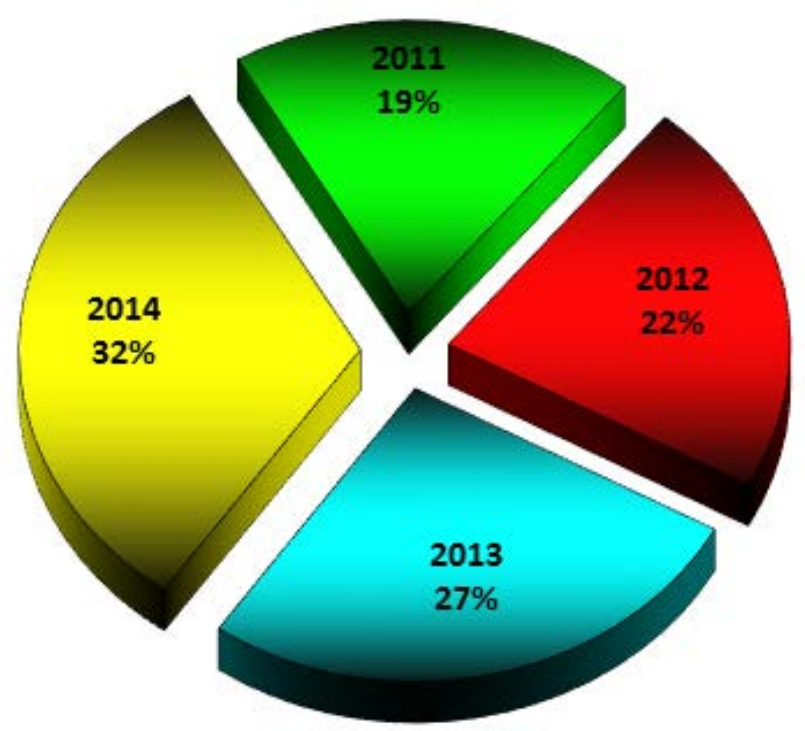

Figure 1 - Distribution of patients on years follows:

The distribution of patients on gender was as
» Year 2011

»Year 2012

» Year 2013

» Year 2014

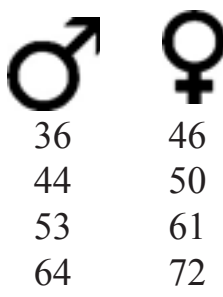

From a total of 426 patients diagnosed with basocellular carcinoma located in the facial region, post excisional defects were resolved using nonmicrosurgical reconstructive procedures, to a number of 96 patients after local dissection could be achieved directly suture, to a number of 208 patients was used full thickness free skin plasty and a total of 120 patients were used local flaps as reconstruction procedure, in plastic surgery clinic and reconstructive Microsurgery of the Constanta County Emergency Hospital.

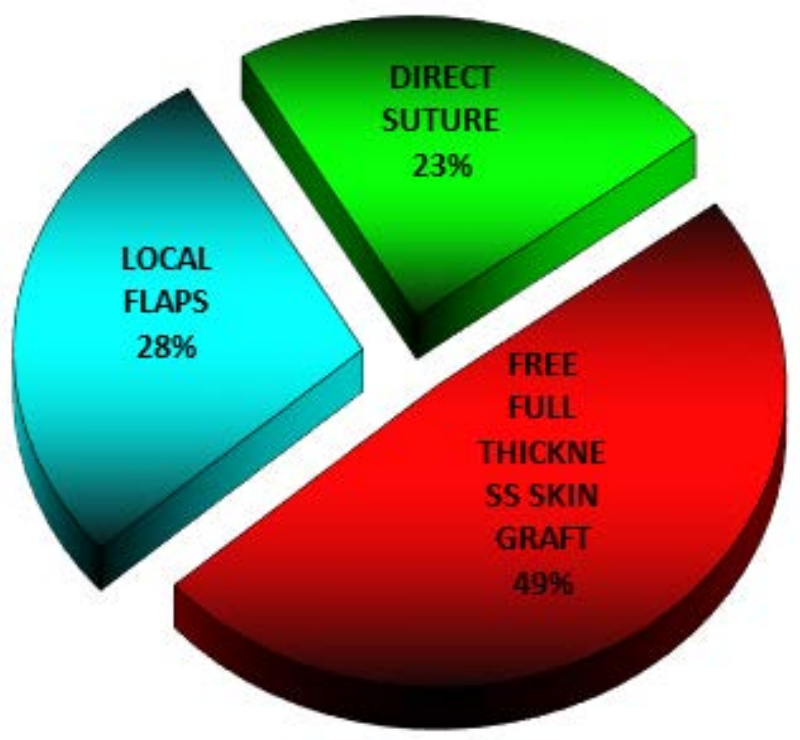

Figure 3 - Methods used

\section{Clinical Cases}

I. Old female patient of 68 years with a basocellular carcinoma in the left wing of the nose region.

Figure 2 - Gender distribution of patients 


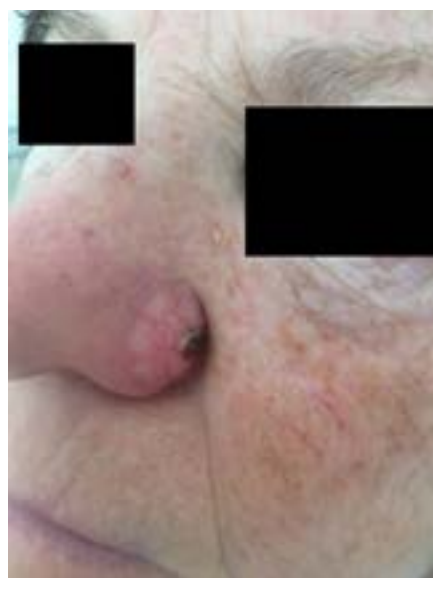

Figure 4 - Preoperative aspect.

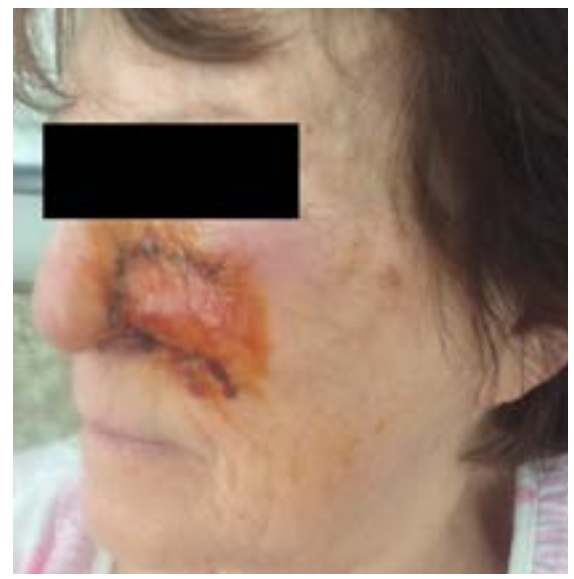

Figure 5 - Postoperatively aspect at 24 hours.

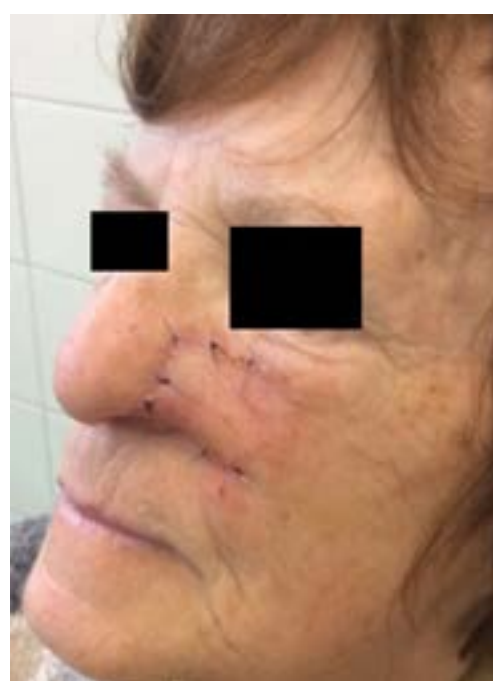

Figure 6 - Postoperatively aspect at 12 days.
It required local flap reconstruction because deep excision let exposed the nasal follicles bulbs risk of necrosis of the wall of the nose.

II. Male patient aged 41 years with a basocellular carcinoma in the internal angle of the right eye.
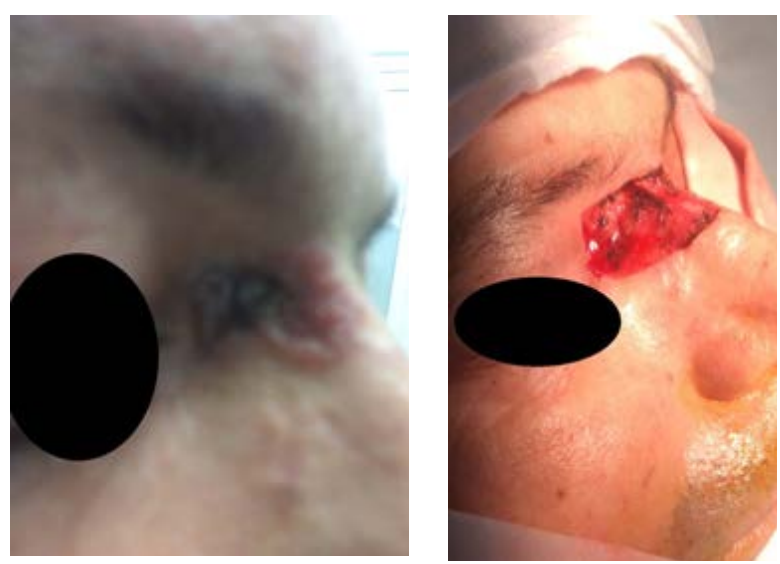

Figure 7-Preoperative aspect. Figure 8 - Intraoperative aspect.

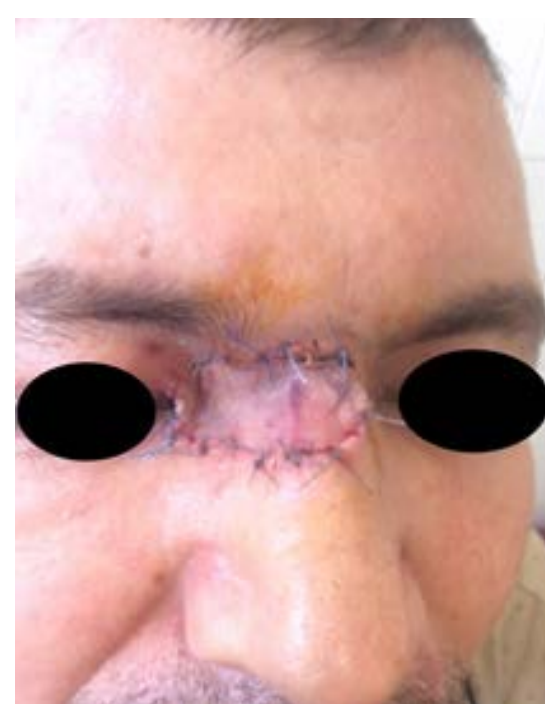

Figure 9 - Postoperatively aspect at 12 days. 


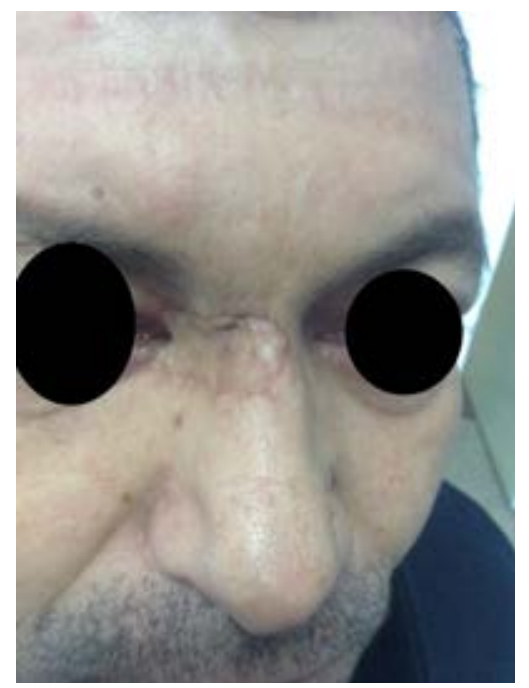

Figure 10 Postoperatively aspect at one month.

Relapsed carcinoma (cauterized to dermatovenerology) in which case the clinic protocol requires repair with autologous full thickness free skin transplant.

III. Female patient aged 77 years with a basocellular carcinoma in the internal angle of the right eye.

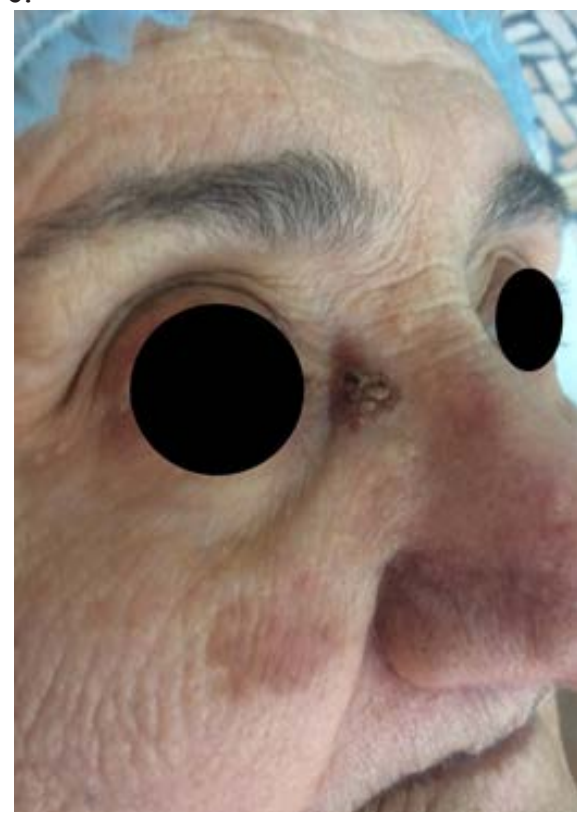

Figure 11 - Preoperative aspect.

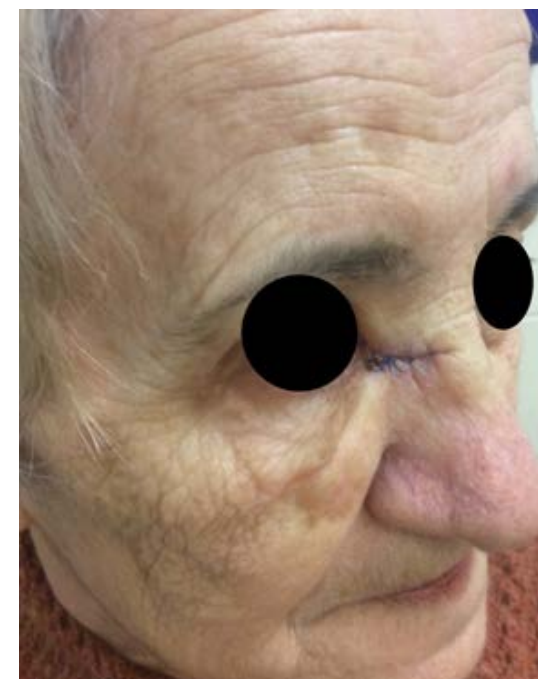

Figure 12 - Postoperatively aspect at 12 days.

Safety marginal excisional 5-6 mm carcinoma; after local dissection can be achieved directly suture.

In all presented cases histopathology examination revealed correct excision marginal and in depth.

\section{Conclusions}

- the retrospective study outstanding at a significant growing up - even worrying - of the incidence of basocellular carcinomas of the face - the percentage reaching $32 \%$ in 2014 to $19 \%$ in 2011 , of all patients take in the retrospective study conducted 2011-2014.

- facial defects reconstruction after excision of basocellular carcinomas can be done in an overwhelming percentage by non-microsurgical procedures.

- in the present study full-thickness autologous free skin transplant accounted for $49 \%$ of casuistry; regional flaps were used in a $28 \%$ and direct suture at a rate of $23 \%$.

- from aesthetically consistently positive results were obtained using the procedures mentioned 
above and should be emphasized, the best results are obtained when direct suture of the defect edges can be achieved this are obviously conditioned by early addressing of the patient.

- chose of reconstruction procedures after excision of facial basocellular carcinomas must be one eclectic in that it is necessary to consider a number of variables: the facial area affected, the patient's age, the extent of the post excisional defect and not least the context etiopathology (recurrent carcinomas, many recurrent and/or irradiated).

\section{References}

1. Achauer, M.B. \& Eriksson, E. (2000). Plastic Surgery: indications, operations and outcomes, St Louis (MO): Mosby

2. McCarthy, J.G., Mai, J.W. \& Littler, J.W (1990). Plastic Surgery. Philadelphia: Sounders Company

3. Lascar, I. (sub redactie). (2005). Principii de chirurgie plastica si microchirurgie reconstructiva. Bucuresti: Ed. National

4. Gheorghita, C. (2014). Modalitati de reconstructie microchirurgicala a fetei dupa rezectii oncologice. Bucuresti: Teza de doctorat - UMF Carol Davila,

5. Lascar,I.(subredactie).(2008). Tratatdechirurgie plastica si microchirurgie reconstructive. Volum 4. Bucuresti: Ed.Academiei Romane. 